\title{
Chemotherapy-Induced Peripheral Neuropathy in a Subpopulation of Mexican Pediatric Patients with Acute Lymphoblastic Leukemia
}

\author{
Irving Jesus Vivas-Rosales, ${ }^{1}$ Liliana Garcia-Saavedra, ${ }^{2}$ Jorge Alfonso Martin-Trejo, ${ }^{3}$ Juan Manuel \\ Mejia-Arangure, ${ }^{4}$ Nora Patricia Victorio-Garcia, ${ }^{5}$ Alejandro Herrera-Landero, ${ }^{6}$ and Juan Carlos \\ Núñez-Enríquez ${ }^{7, *}$ \\ ${ }^{1}$ Department of Pediatric, Pediatrics Hospital, National Medical Center (The Siglo XXI), Mexican Institute of Social Security \\ ${ }^{2}$ Department of Physical Medicine and Rehabilitation, Pediatrics Hospital, National Medical Center (The Siglo XXI), Mexican Institute of Social Security \\ ${ }^{3}$ Department of Hematology, Pediatrics Hospital, National Medical Center (The Siglo XXI), Mexican Institute of Social Security \\ ${ }^{4}$ Division of Evaluation of Research, Health Research Coordination, Mexican Institute of Social Security \\ ${ }^{5}$ Faculty of Medicine of Tampico “Dr. Alberto Romo Caballero", Autonomous University of Tamaulipas \\ ${ }^{6}$ Traumatology and Orthopedics Hospital “Lomas Verdes" Mexican Institute of Social Security \\ ${ }^{7}$ Clinical Epidemiology Research Department, Pediatrics Hospital, National Medical Center (The Siglo XXI), Mexican Institute of Social Security
}

"Corresponding author: Dr Juan Carlos Núñez-Enríquez, Clinical Epmidemiology Research Department, Pediatrics Hospital, The Siglo XXI National Medical Center, Mexican Institute of Social Security, Avenida Cuauhtemoc 330, Col. Doctores, Delegacion Cuauhtemoc, Mexico City, Mexico. Tel: +52-5556276900, E-mail: jcarlos_nu@hotmail.com

Received 2017 April 18; Revised 2017 June 24; Accepted 2017 July 20.

\begin{abstract}
Background: Chemotherapy-induced peripheral neuropathy (CIPN) is frequent in children with acute lymphoblastic leukemia (ALL). Neurological manifestations can be grouped into one of the three functional divisions of the peripheral nervous system (PNS): sensory, motor and autonomic. One of the chemotherapeutic agents used in ALL is vincristine which has been associated with neuropathy in these children. This type of neuropathy can be transient but also leave permanent sequels that decrease patient's quality of life.

Objectives: To know the frequency and type of neuropathy induced by chemotherapy in a subpopulation of Mexican pediatric patients with acute lymphoblastic leukemia.

Methods: A cross-sectional study was conducted. There were included all pediatric patients with ALL diagnosed de novo from 2010 to December 31, 2013 who met the selection criteria. Descriptive statistics were used to determine the frequency and type of CIPN and information was described according to different clinical variables, type of treatment, risk classification, and ALL subtype according to the immunophenotype.

Results: A total of 32 patients with acute lymphoblastic leukemia were included, mainly of early pre-B immunophenotype (93.8\%), being $59.4 \%$ classified as high risk patients at the time of diagnosis. CIPN of sensory type was $78.1 \%$, motor $34.3 \%$ and autonomic 40.6\%. A significant proportion of patients (46.9\%) were examined during maintenance phase.

Conclusions: To our knowledge, this is the first study to report the frequency and type of CIPN in a subpopulation of Mexican children with acute lymphoblastic leukemia. Frequency of CIPN was higher than that reported in other populations. By considering that prognosis of a patient presenting CIPN could be considered as favorable after treatment suspension (in most cases), it is not always reversible and affects patient's quality of life. Therefore, it is necessary for all patients with ALL to be periodically monitored during treatment through neurological examination for detecting and immediately initiating their rehabilitation treatment.
\end{abstract}

Keywords: Antineoplastic Agents, Children, Leukemia, Peripheral Nervous System Diseases

\section{Background}

Leukemia is the most common type of neoplasia in children aged 0 - 14 years $(1,2)$. Acute lymphoblastic leukemia (ALL) is the most common subtype of leukemia. Treatment for ALL depends almost entirely on the chemotherapy regimen. In recent years, long-term complications secondary to treatment have been reported to be more frequent due to an increase in ALL survival rates.

Chemotherapy-induced peripheral neuropathy (CIPN) is defined as a lesion, inflammation, or degeneration of the peripheral nerves caused by the administration of a chemotherapeutic agent (3). Neurological manifestations can be grouped into one of the three functional divisions of the peripheral nervous system(PNS): sensory, motor and autonomic. The CIPN usually presents in nerves of greater length, that is why, the first symptoms occur in feet and hands, and progresses from distal to proximal (4).

Currently, the incidence of CIPN is 30\% - 40\% for all types of cancer. CIPN affects not only the quality of life but 
also, it can lead to interruption or decrease in the dose of chemotherapy which could potentially affect the overall survival of cancer patients (5-7).

Antineoplastic agents most frequently associated with peripheral neuropathy are taxanes, platines, vincristine, thalidomide and bortezomib $(8,9)$. The degree and type of neuropathy depends on the chemotherapeutic agent employed, as well as its cumulative dose. The diagnosis of CIPN is established by abnormalities in the medical history, physical examination, and nerve electrophysiology (10).

Noteworthy, so far no drug or nutritional supplement has been shown to be capable of preventing or limiting chemotherapy-induced neuropathy, highlighting the relevance to opportunely detect and treat this complication in children with leukemia (11). To our knowledge, there is no previous report on the frequency and type of CIPN in Mexican children with ALL.

\section{Objectives}

To report the frequency and type of chemotherapyinduced peripheral neuropathy in a sample of acute lymphoblastic leukemia in Mexican pediatric patients.

\section{Methods}

A descriptive cross-sectional study was conducted. We included all children with diagnosis of ALL who met the selection criteria and attended during study period (January 1st to July 30th, 2016) at either outpatient clinic or hospitalization department of the Pediatrics Hospital of the National Medical Center (The Siglo XXI), Mexican Institute of Social Security, which is a tertiary referral hospital located in Mexico City. Study was approved by the local scientific research and ethics committee with the number: R-20153603-77. Informed consent was obtained from child's parents.

\subsection{Selection Criteria}

Inclusion criteria: Patients diagnosed with acute lymphoblastic leukemia aged $>5$ years. Exclusion/elimination criteria: ALL in Down syndrome patients, patients with psychomotor retardation, cerebral palsy, being under effects of sedation and on treatment with neuropathic drugs, patients who were in intensive care, intubated, or who were not in clinical conditions to perform the neurological examination, those with any neoplasia prior to diagnosis of ALL, patients who required bone marrow transplantation, having any electrolyte disturbances during the evaluation, with altered state of consciousness, and patients with altered muscle tone, muscle stretching reflexes, in the superficial and deep sensitivity initially diagnosed, were excluded from the study. Patients who accepted to participate in the study then decided not to continue, and patients in whom neurological examination was not completed, were also excluded.

The following information was collected from clinical charts: sex, age at diagnosis, FAB classification (L1, L2, L3), risk classification (standard, intermediate, high), immunophenotype (pro-B, pre-B, mature B-cell, T-lineage, biphenotypic), chemotherapy scheme used according to the risk of ALL, phase of chemotherapy at the time of neurological examination (remission induction, consolidation, maintenance, reinduction, palliative, surveillance), relapse in the patient, site of relapse.

Chemotherapy-induced peripheral neuropathy was assessed according to ped-mTNS criteria and looking for detecting sensory, motor, combined neuropathy (12). Autonomic system was examined by asking questions about symptoms as paresthesias, muscle weakness, dizziness and lypothymia; and through the evaluation of osteotendinous reflexes. These parameters are part of the validated pediatric modified scale of chemotherapy-induced neuropathy (ped-mTNS). Muscle strength was assessed by using ped-mTNS muscular strength items. Distal muscular strength was assessed using Daniel's scale through manual muscle examination in interossei, wrist extensors, extensor digitorum longus and ankle dorsiflexors (13).

Data were analyzed using SPSS software version 21 . Descriptive analysis was performed according to the scale of measurement of the variables, using measures of central tendency and dispersion. For qualitative variables, simple frequencies and percentages were calculated. For quantitative variables it was determined whether the distribution was parametric or non-parametric. In case of parametric distribution, we calculated means and standard deviations, in case they did not have parametric distribution we used median and interquartile ranges.

\section{Results}

A total of 32 pediatric patients diagnosed with acute lymphoblastic leukemia (ALL) were included in the present study, from which $53.1 \%$ were females with a mean age of 9.7 years ( $\mathrm{SD} \pm 3.1$ ). According to the morphological classification of leukemia (FAB classification), $84.4 \%$ corresponded to L1 ALL, by immunophenotype, 93.8\% were found to have an early pre-B type immunophenotype, regarding risk classification, $59.4 \%$ were high risk of relapse patients. Noteworthy, $46.9 \%$ of the study population was in the maintenance phase. Relapse patients were included, 
Items Included in the ped-mTNS to Assess Muscle Strength

Muscle assessed: right/left

Measured muscle level.

Big toe - -

Ankle -/-

Abduction of phalanges $\%$

Wrist extension $-/$

0. Absence of contraction (Grade 0 Daniels scale: absence of contraction)

1. Contraction without movements (Grade 1 Daniels scale: visible or palpable muscle contraction)

2. Movement that does not overcome gravity (Grade 2 Daniels scale: contraction in the horizontal plane that does not overcome gravity).

3. Complete movement that overcomes gravity (Grade 3 Daniels scale: complete movement that overcomes gravity without resistance)

4. Movement with partial resistance (Grade 4 Daniels scale: movement with partial resistance).

5. Movement with maximum resistance (Grade 5 Daniels scale: Movement with maximum resistance).

${ }^{a}$ Rating scale from 0 to 5 (0: no contraction, 1: visible contraction but immovable, 2: movable without gravity but immovable against it, 3: movable against gravity, 4 : slight decrease, 5: normal).

bone marrow relapse being the most frequent type (Table 1).

Table 2 lists the drugs most commonly used in our study. Patients with relapse and palliative chemotherapy had received more doses of chemotherapy, generally by cycles of re-induction to remission. Table 2 lists the cumulative dose for each of the drugs used during each chemotherapy phase, based on the protocol HP 09 for Acute lymphoblastic leukemia, pediatrics hospital of national medical center (The Siglo XXI) (protocol in force).

As to the frequency of chemotherapy-induced neuropathy, in our study we found that by physical examination, $78.1 \%$ had a sensitivity alteration, 34.3\% in motor exploration and $40.6 \%$ autonomic alteration (Table 3 ). Type of neuropathy was divided according to the subtype of affection of the peripheral nervous system (sensory, autonomic, and motor type). No statistically significant difference was found with regard to gender (male/female) for any type of neuropathy ( $\mathrm{P}>0.05)$.

From the findings at interrogation, $12.5 \%$ reported parestesias at some time during or after chemotherapy. 6.3\% had problems to button their shirt, both patients were in maintenance phase. $6.3 \%$ referred problems with walking; both patients were in the maintenance phase. $12.5 \%$ of the patients reported having problems when going up or down the stairs. In addition, $12.5 \%$ felt dizzy when getting out of bed (Table 4 ).

Regarding the findings in physical examination, only one patient presented alteration to the light touch at the level of the toes. In the exploration $78.1 \%$ had alterations in vibratory sensitivity, 34.3\% were found with an alteration in right ankle muscle strength. On the contralateral side, $34.3 \%$ presented alteration in strength similar to the right limb. The abduction muscle strength of the right phalanges was altered in $21.9 \%$ of the total patients. The distribution and percentages were similar in the exploration of muscle strength for abduction of the left phalanges. Muscle strength for right wrist extension was altered in $21.9 \%$ of the total patients. The findings were similar to the physical examination of muscle strength for the left wrist extension. The exploration of the right Achilles reflex was altered in $40.6 \%$. The percentages and distribution were similar for the deep left Achilles reflex. In the right patellar reflex, $18.8 \%$ were altered. The percentages and distribution for chemotherapy were similar for the left patellar reflex (Table 4).

\section{Discussion}

The present study has allowed us to describe for the first time the type and frequency of chemotherapyinduced peripheral neuropathy in pediatric patients with acute lymphoblastic leukemia treated at a Mexican third level hospital.

Ramcharden et al. found in pediatric survivors of leukemia who had no subjective symptoms of neuropathy despite having an exploration or nerve conduction studies altered (14). In the present study, we observed that chemotherapy-induced peripheral neuropathy can be found even before the end of treatment. Thus, compared 
Table 1. Clinical Characteristics of ALL Children Included in Present Study

\begin{tabular}{|c|c|c|}
\hline Clinical Variables & $\mathbf{N}=\mathbf{3 2}$ & $\%$ \\
\hline \multicolumn{3}{|l|}{ Sex } \\
\hline Male & 15 & 46.9 \\
\hline Female & 17 & 53.1 \\
\hline \multicolumn{3}{|l|}{ FAB classification } \\
\hline L1 & 27 & 84.4 \\
\hline L2 & 5 & 15.6 \\
\hline \multicolumn{3}{|l|}{ Immunophenotype } \\
\hline Early Pre-B & 30 & 93.8 \\
\hline T-lineage & 1 & 3.1 \\
\hline Biphenotypic & 1 & 3.1 \\
\hline \multicolumn{3}{|l|}{ Risk classification } \\
\hline Standard-risk & 10 & 31.3 \\
\hline Intermediate-risk & 3 & 9.4 \\
\hline High-risk & 19 & 59.4 \\
\hline \multicolumn{3}{|l|}{ Chemotherapy phase } \\
\hline Remission induction & 4 & 12.5 \\
\hline Consolidation & 4 & 12.5 \\
\hline Maintenance & 15 & 46.9 \\
\hline Surveillance & 4 & 12.5 \\
\hline Palliative chemotherapy & 1 & 3.1 \\
\hline Relapses & 4 & 12.5 \\
\hline \multicolumn{3}{|l|}{ Sites of relapse } \\
\hline Bone marrow & 2 & 6.2 \\
\hline Bone marrow and testicles & 1 & 3.1 \\
\hline Bone marrow and CNS & 1 & 3.1 \\
\hline
\end{tabular}

to the findings reported by Ramchandren et al., we found nerve alterations up to almost $80 \%$ versus $29.7 \%$ by these authors.

Regarding the cumulative dose, it has been established that dosages of $1.4 \mathrm{mg} / \mathrm{m}^{2}$ dose of vincristine may be safe (15-17). However, most of our patients have cumulative dose of this drug, which has been linked to the risk of chemotherapy-induced neuropathy. Another example, is methotrexate, which shows that high doses, greater than $1.5 \mathrm{~g} / \mathrm{m}^{2}$ is related to adverse effects (18). Some of the chemotherapeutic agents established in therapy for ALL such as ARA-C, have yet to establish the cumulative doses for risk of peripheral neurotoxicity $(19,20)$.

Martinez-Cayuelas et al., reported the frequency of neuropathy during the different phases of leukemia treatment and after the end of it. They observed that most of the neurological complications were presented in the induction phase (43\%), consolidation (17\%) or maintenance $(13 \%)$, and only a low percentage occurred at the beginning of the disease, after treatment and in palliative care phase (20). In our series, 4 patients (12.5\%) reported paresthesias, one patient in the consolidation phase and 3 in the maintenance phase. In addition, 4 patients (12.5\%) reported motor type problems, one in consolidation, two in maintenance and another with relapse in maintenance phase. Regarding physical examination, 25 individuals (71.8\%) presented alteration in vibratory sensitivity. This alteration was greater in maintenance phase with a number of 15 individuals (48.6\%) of our series, unlike what these authors found where the greatest affection was given in the induction phase in $43 \%$ of the cases versus 4 patients (12.5\%) in our study. However, this may be affected by the size of our sample, since the largest population was in the maintenance phase, constituting a number of 15 individuals (46.9\%) against 4 individuals (12.5\%) in the remission induction phase in our series.

Of the neuropathies found by Martinez-Cayuelas et al., $31 \%$ of the population reported pain and weakness of the lower limbs. In our series, the major alteration was of a vibratory type with affection in 25 (80\%) patients of the population. Although vibratory sensitivity is not referred to as pain or weakness, these sensory and motor alterations respectively, correspond to a type of proprioceptive alteration that is also part of the peripheral nervous system and is included in the sensory neuropathy, therefore, the findings are similar to other studies constituting peripheral neuropathy the main condition (21-23). In our series, in addition to the physical examination, reference was made to neuropathy symptoms, this way, motor-type problems were reported in 4 (12.5\%) individuals of our population, and $4(12.5 \%)$ patients reported paresthesias, this is different to the findings of Martinez-Cayuelas et al., where this type of alteration was reported in approximately $30 \%$ of patients. This could be explained by the size of our sample, which is smaller but the findings of sensory and motor alteration are the main ones in both series.

In 2010, Velasco R. and Bruna J., in a review article, commened that chemotherapy-induced peripheral neuropathy is the most frequent neurological complication of cancer treatment, affecting approximately one-third of patients (24). This is similar to the findings by E. MartinezCayuelas, et al., where $30 \%$ of their patients had sensory and motor type complications (18). In our study, in a sample of 32 patients, the distribution was approximately 25 (80\%) patients with a sensory type, 11 (34\%) patients with a motor type and 13 (40\%) individuals with autonomic type. This shows that the prevalence of chemotherapy-induced neuropathy in our patients is higher than that reported 
Table 2. Cumulative Dose of Chemotherapy Agents on Different Phases of Chemotherapy According to the HP 09 ALL Protocol ${ }^{\mathrm{a}}$

\begin{tabular}{|c|c|c|c|c|c|c|}
\hline \multirow[t]{2}{*}{ Chemotherapy Agent } & \multicolumn{6}{|c|}{ Chemotherapy Phase And Cumulative Doses Received } \\
\hline & Induction to remission & Consolidation & Maintenance & Surveillance & Relapse & Palliative chemotherapy \\
\hline Vincristine, $\mathbf{m g} / \mathbf{m}^{2}$ & 6 & 9 & 15 & 15 & 21 & 21 \\
\hline Daunorubicin, $\mathrm{mg} / \mathrm{m}^{2}$ & 120 & 180 & 300 & 300 & 420 & 420 \\
\hline L-Asparaginase, $\mathrm{UI} / \mathrm{m}^{2}$ & 40,000 & 115,000 & 190,000 & 190,000 & 210,000 & 210,000 \\
\hline Cyclophosphamide, $\mathrm{mg} / \mathrm{m}^{2}$ & 0 & 200,000 & 2000 & 2000 & 2000 & 2000 \\
\hline VP-16, $\mathrm{mg} / \mathrm{m}^{2}$ & 0 & 600 & 600 & 600 & 600 & 600 \\
\hline Ifosfamide, $\mathrm{mg} / \mathrm{m}^{2}$ & 0 & 5000 & 5000 & 5000 & 5000 & 6500 \\
\hline Methotrexate, $\mathrm{mg} / \mathrm{m}^{2}$ & 0 & 6000 & 6000 & 6000 & 6000 & 6000 \\
\hline
\end{tabular}

${ }^{\text {a }}$ shows shows the cumulative dose on each Chemotherapy phase according to the HP 09 ALL protocol.

Table 3. Frequency and Types of Chemotherapy-Induced Peripheral Neuropathy in Study Population

\begin{tabular}{|ccc|}
\hline Neuropathy & $\mathbf{N}=\mathbf{3 2}$ & $\%$ \\
\hline By interrogation & & \\
\hline Sensory & 4 & 12.5 \\
\hline Motor & 4 & 12.5 \\
\hline Autonomic & 4 & 12.5 \\
\hline By physical examination & & \\
\hline Sensory & 25 & 78.1 \\
\hline Motor & 11 & 34.3 \\
\hline Autonomic & 13 & 40.6 \\
\hline
\end{tabular}

in other studies, even when the chemotherapy phases in which these alterations were detected are taken into account. We do not know why this higher frequency of neuropathy since chemotherapy doses adjusted to the weight of the patient were used.

In studies that have been conducted to measure chemotherapy-induced neuropathy in children with acute lymphoblastic leukemia, the most frequent cause has been vincristine treatment, which turned out to be dose dependent, and in which it has been observed that most of these complications have been found two weeks after administration of vincristine (17) Lavoie Smith et al, used the Neuropathy score pediatric vincristine scale in a total of 128 patients, receiving $1.5 \mathrm{mg} / \mathrm{m}^{2}$ of vincristine, found a $78 \%$ of peripheral neuropathy (25) which is exactly similar to the finding observed in present study.

\subsection{Study Limitations}

One of the limitations of our study is the research design since it is a cross-sectional descriptive study in which it was not possible to determine if these patients already had neuropathy at the time of the exploration since previous measurements had not been made. Other limitation could be the small sample size of ALL patients examined; however, we included all those patients attended during study period at outpatient clinic and/or during hospitalization and who met selection criteria. We consider that the next step is to design multicenter, prospective research studies that include a larger sample size in order to determine the prevalence of chemotherapy-induced neuropathy in our population.

Moreover, we were not able to establish what type of neurotoxic agent was responsible for the neuropathy. It is well known that corticosteroids may cause weakness and induce myopathy and this could have affected our results. It is important to highlight that all patients in present study daily received as part of their chemotherapy treatment a systemic steroid at a high dose during the first 4 weeks (phase of remission induction). However, there are two important issues that have to be taken into consideration: first, only 4 (12.5\%) patients were examined during this phase of treatment (induction to remission phase), being the majority of patients in phases of treatment where systemic steroids are not used; and second, it should be noted that the evaluation of muscle strength in our study was based mainly on distal musculature and not on proximal, which according to the literature characterize the steroid-induced myopathy (26).

Another possible limitation of our study may have been the failure to confirm peripheral neuropathy with electrodiagnostic studies. However, until date there is no universally accepted tool for diagnosing chemotherapyinduced peripheral neuropathy in children and several scales (including or not electrodiagnostic studies) have been used to detect this complication in children receiving chemotherapy $(12,27,28)$. Some examples are: the total 
neuropathy scale (TNS), clinical neuropathy scale, and the modified neuropathy scale. Recently, the modified pediatric scale for total neuropathy (ped-mTNS) was validated as a variant of TNS (for not including electrodiagnostic studies), and has been considered as a reliable and valid measure for detecting CIPN in children with cancer and leukemia $(27,28)$. Moreover, it has been also reported that sensitivity to detect CIPN with this scale is greater than with previous ones. For this reason, we used in present research the Ped-mTNS wich consisted of three sets of questions about sensory symptoms, pain, motor function and autonomic function, as well as a five-part neurological examination that includes light touch explored by monofilament, biotensor vibration, pin-prick sensitivity, distal force through manual muscle examination (performed on interossei, wrist extensors, extensor digitorum muscle and ankle dorsiflexors), and deep tendon reflexes $(27,28)$.

\subsection{Conclusions}

To our knowledge, this is the first study to report the frequency and type of CIPN in a subpopulation of Mexican children with acute lymphoblastic leukemia. Frequency of CIPN was higher than that reported in other populations. By considering that prognosis of a patient presenting CIPN could be considered as favorable after treatment suspension (in most cases), it is not always reversible and affects patient's quality of life. Therefore, it is necessary for all patients with ALL to be periodically monitored during treatment through neurological examination for detecting and immediately initiating their rehabilitation treatment.

\section{Acknowledgments}

We thank the hematology service and pediatric hematologists Dr. Maria del Carmen Rodriguez Zepeda, Dr. Jorge Alfonso Martin Trejo, Dr. Karina A. Solis Labastida, Dr. Benito Bautista Hernandez, the residents of hematology, and the social worker (Lulu) of the Pediatric Hospital of the National Medical Center Siglo XXI, IMSS who all contributed with granting the facilities so that the student of Pediatrics and the Service of Rehabilitation could carry out the physical examination and the interrogation of the patients.

\section{References}

1. Fajardo-Gutierrez A, Mejía-Aranguré M, Gómez-Delgado A. Epidemiología de las neoplasias malignas en ni-os residentes del Distrito Federal (1982-1991) [In Spanish]. Bol Med Hosp Infant Mex. 1995;52:507-16.

2. Fajardo-Gutierrez A, Mejia-Arangure JM, Hernandez-Cruz L, MendozaSanchez HF, Garduno-Espinosa J, Martinez-Garcia MC. [Descriptive epidemiology of malignant tumors in children]. Rev Panam Salud Publica. 1999;6(2):75-88. [PubMed:10574008].
3. Armstrong T, Almadrones L, Gilbert MR. Chemotherapy-induced peripheral neuropathy. Oncol Nurs Forum. 2005;32(2):305-11. doi 10.1188/05.ONF.305-311. [PubMed: 15759068].

4. Gilchrist L. Chemotherapy-induced peripheral neuropathy in pediatric cancer patients. Semin Pediatr Neurol. 2012;19(1):9-17. doi 10.1016/j.spen.2012.02.011. [PubMed: 22641071].

5. Cavaletti G, Bogliun G, Marzorati L, Tredici G, Colombo N, Parma $\mathrm{G}$, et al. Long-term peripheral neurotoxicity of cisplatin in patients with successfully treated epithelial ovarian cancer. Anticancer Res. 1994;14(3B):1287-92. [PubMed: 8067698].

6. Wolf S, Barton D, Kottschade L, Grothey A, Loprinzi C. Chemotherapyinduced peripheral neuropathy: prevention and treatment strategies. Eur J Cancer. 2008;44(11):1507-15. doi: 10.1016/j.ejca.2008.04.018. [PubMed: 18571399].

7. Mileshkin L, Stark R, Day B, Seymour JF, Zeldis JB, Prince HM Development of neuropathy in patients with myeloma treated with thalidomide: patterns of occurrence and the role of electrophysiologic monitoring. J Clin Oncol. 2006;24(27):4507-14. doi 10.1200/JCO.2006.05.6689. [PubMed: 16940275].

8. Cardona AF, Ortiz LD, Reveiz L. Neuropatía inducida por el tratamiento médico del cáncer. Med UIS. 2010;23(2):103-27.

9. Argyriou AA, Bruna J, Marmiroli P, Cavaletti G. Chemotherapyinduced peripheral neurotoxicity (CIPN): an update. Crit Rev Oncol Hematol. 2012;82(1):51-77. doi: 10.1016/j.critrevonc.2011.04.012. [PubMed: 21908200].

10. England JD, Gronseth GS, Franklin G, Miller RG, Asbury AK, Carter GT, et al. Distal symmetric polyneuropathy: a definition for clinical research: report of the American Academy of Neurology, the American Association of Electrodiagnostic Medicine, and the American Academy of Physical Medicine and Rehabilitation. Neurology. 2005;64(2):199-207. doi: 10.1212/01.WNL.0000149522.32823.EA. [PubMed: 15668414].

11. Albers J, Chaudhry V, Cavaletti G, Donehower R. Interventions for preventing neuropathy caused by cisplatin and related compounds. Cochrane Database Syst Rev. 2007(1):CD005228. doi 10.1002/14651858.CD005228.pub2. [PubMed: 17253547].

12. Gilchrist LS, Tanner L. The pediatric-modified total neuropathy score: a reliable and valid measure of chemotherapy-induced peripheral neuropathy in children with non-CNS cancers. Support Care Cancer. 2013;21(3):847-56. doi: 10.1007/s00520-012-1591-8. [PubMed: 22993026].

13. Hislop HJ, Montgomery J. Daniels and Worthingham's Muscle Testing: Techniques of Manual Examination. 6 ed. Philadelphia: Saunders; 1995. pp. 2-6.

14. Ramchandren S, Leonard M, Mody RJ, Donohue JE, Moyer J, Hutchinson R, et al. Peripheral neuropathy in survivors of childhood acute lymphoblastic leukemia. J Peripher Nerv Syst. 2009;14(3):184-9. doi: 10.1111/j.1529-8027.2009.00230.x. [PubMed: 19909482].

15. Legha SS. Vincristine neurotoxicity. Pathophysiology and management. Med Toxicol. 1986;1(6):421-7. doi:10.1007/BF03259853. [PubMed 3540519].

16. Sandler SG, Tobin W, Henderson ES. Vincristine-induced neuropathy. A clinical study of fifty leukemic patients. Neurology. 1969;19(4):36774. [PubMed: 5813374].

17. Díaz-Jaimes E, Pe-aloza-Ochoa L, Parada-Onoko PM. Electrophysiolog ical changes of peripheral neuropathy with vincristine after a physical therapy program in pediatric patients with acute lymphoblastic leukemia. Bol Med Hosp Infant Mex. 2009;66(6):529-36.

18. De Braganca KC, Packer RJ. Neurotoxicity of chemotherapeutic and biologic agents in children with cancer. Curr Neurol Neurosci Rep. 2008;8(2):114-22. [PubMed: 18460279].

19. Openshaw H, Slatkin NE, Stein AS, Hinton DR, Forman SJ. Acute polyneuropathy after high dose cytosine arabinoside in patients with leukemia. Cancer. 1996;78(9):1899-905. [PubMed: 8909309]. 
20. Martinez-Cayuelas E, Domingo-Jimenez R, Pascual-Gazquez JF, Martinez-Salcedo E, Alarcon-Martinez H, Bermudez-Cortes M, et al. [Neurological complications in the population of children with leukaemia]. Rev Neurol. 2015;60(3):108-14. [PubMed: 25624086].

21. Vagace JM, de la Maya MD, Caceres-Marzal C, Gonzalez de Murillo S, Gervasini G. Central nervous system chemotoxicity during treatment of pediatric acute lymphoblastic leukemia/lymphoma. Crit Rev Oncol Hematol. 2012;84(2):274-86. doi: 10.1016/j.critrevonc.2012.04.003. [PubMed: 22578745].

22. Lo Nigro L, Di Cataldo A, Schiliro G. Acute neurotoxicity in children with B-lineage acute lymphoblastic leukemia (B-ALL) treated with intermediate risk protocols. Med Pediatr Oncol. 2000;35(5):449-55. [PubMed: 11070476].

23. Parasole R, Petruzziello F, Menna G, Mangione A, Cianciulli E, Buffardi $S$, et al. Central nervous system complications during treatment of acute lymphoblastic leukemia in a single pediatric institution. Leuk Lymphoma. 2010;51(6):1063-71. doi: 10.3109/10428191003754608.
[PubMed: 20470218].

24. Velasco R, Bruna J. [Chemotherapy-induced peripheral neuropathy: an unresolved issue]. Neurologia. 2010;25(2):116-31. doi: 10.1016/S02134853(10)70036-0. [PubMed: 20487712].

25. Lavoie Smith EM, Li L, Chiang C, Thomas K, Hutchinson RJ, Wells EM, et al. Patterns and severity of vincristine-induced peripheral neuropathy in children with acute lymphoblastic leukemia. J Peripher Nerv Syst. 2015;20(1):37-46. doi: 10.1111/jns.12114. [PubMed: 25977177].

26. Perrot S, Le Jeunne C. [Steroid-induced myopathy]. Presse Med. 2012;41(4):422-6. doi:10.1016/j.lpm.2012.01.004. [PubMed: 22326665].

27. Farquhar-Smith P. Chemotherapy-induced neuropathic pain. Curr Opin Support Palliat Care. 2011;5(1):1-7. doi: 10.1097/SPC.0b013e328342f9cc. [PubMed: 21192267].

28. Gilchrist LS, Marais L, Tanner L. Comparison of two chemotherapyinduced peripheral neuropathy measurement approaches in children. Support Care Cancer. 2014;22(2):359-66. doi:10.1007/s00520-0131981-6. [PubMed: 24072474]. 
Table 4. Frequency and Types of Neuropathic Alterations According to Chemotherapy Phase When They Were Examined

\begin{tabular}{|c|c|c|c|c|c|c|c|c|c|c|c|c|c|c|}
\hline \multirow{3}{*}{$\begin{array}{l}\text { Findings } \\
\text { Finding in the directed } \\
\text { interrogation }\end{array}$} & \multicolumn{2}{|c|}{ Total Population } & \multicolumn{12}{|c|}{ Chemotherapy Phases } \\
\hline & \multirow[b]{2}{*}{$\mathbf{n}=32$} & \multirow[b]{2}{*}{$\%$} & \multicolumn{2}{|c|}{ Remission induction } & \multicolumn{2}{|c|}{ Consolidation } & \multicolumn{2}{|c|}{ Maintenance } & \multicolumn{2}{|c|}{ Surveillance } & \multicolumn{2}{|c|}{ Relapse } & \multicolumn{2}{|c|}{ In palliative CT } \\
\hline & & & $\mathbf{n}=\mathbf{4}$ & $\%$ & $\mathrm{n}=\mathbf{4}$ & $\%$ & $\mathrm{n}=15$ & $\%$ & $\mathrm{n}=4$ & $\%$ & $n=4$ & $\%$ & $\mathbf{n}=\mathbf{1}$ & $\%$ \\
\hline Paresthesia & 4 & 12.5 & - & - & - & - & - & - & - & - & - & - & - & - \\
\hline $\begin{array}{l}\text { Symptoms } \\
\text { limited to the } \\
\text { toes }\end{array}$ & 1 & 3.1 & 0 & 0 & 1 & 100 & 0 & 0 & 0 & 0 & 0 & 0 & 0 & 0 \\
\hline $\begin{array}{l}\text { Symptoms up } \\
\text { to the knee or } \\
\text { elbow }\end{array}$ & 1 & 3.1 & 0 & 0 & 0 & 0 & 1 & 33.3 & 0 & 0 & 0 & 0 & 0 & 0 \\
\hline $\begin{array}{l}\text { Symptoms } \\
\text { above the knee } \\
\text { or elbow }\end{array}$ & 2 & 6.3 & 0 & 0 & 0 & 0 & 2 & 66.6 & 0 & 0 & 0 & 0 & 0 & 0 \\
\hline $\begin{array}{l}\text { Problems to button up } \\
\text { your shirt }\end{array}$ & 2 & 6.3 & - & - & - & - & - & - & - & - & - & - & - & - \\
\hline Needs help & 2 & 6.3 & 0 & 0 & 0 & 0 & 2 & 100 & 0 & 0 & 0 & 0 & 0 & 0 \\
\hline Is walking difficult & 2 & 6.3 & - & - & - & - & - & - & - & - & - & - & - & - \\
\hline Not difficult & 1 & 3.1 & 0 & 0 & 0 & 0 & 1 & 50 & 0 & 0 & 0 & 0 & 0 & 0 \\
\hline Cannot walk & 1 & 3.1 & 0 & 0 & 0 & 0 & 1 & 50 & 0 & 0 & 0 & 0 & 0 & 0 \\
\hline $\begin{array}{l}\text { Problems going up or } \\
\text { down the stairs }\end{array}$ & 4 & 12.5 & - & . & - & - & - & . & - & - & . & . & - & - \\
\hline Not difficult & 1 & 3.1 & 0 & 0 & 1 & 100 & 0 & 0 & 0 & 0 & 0 & 0 & 0 & 0 \\
\hline $\begin{array}{l}\text { Somewhat } \\
\text { difficult }\end{array}$ & 1 & 3.1 & 0 & 0 & 0 & 0 & 0 & 0 & 0 & 0 & 1 & 100 & 0 & 0 \\
\hline Needs help & 1 & 3.1 & 0 & 0 & 0 & 0 & 1 & 50 & 0 & 0 & 0 & 0 & 0 & 0 \\
\hline $\begin{array}{l}\text { Cannot go up or } \\
\text { down the stairs }\end{array}$ & 1 & 3.1 & 0 & 0 & 0 & 0 & 1 & 50 & 0 & 0 & 0 & 0 & 0 & 0 \\
\hline $\begin{array}{l}\text { Feels dizzy when } \\
\text { getting up from bed }\end{array}$ & 4 & 12.5 & - & - & - & - & - & - & - & - & - & $\cdot$ & - & - \\
\hline A little & 3 & 9.4 & 1 & 100 & 0 & 0 & 1 & 50 & 0 & 0 & 1 & 100 & 0 & 0 \\
\hline Almost always & 1 & 3.1 & 0 & 0 & 0 & 0 & 1 & 50 & 0 & 0 & 0 & 0 & 0 & 0 \\
\hline \multicolumn{15}{|l|}{$\begin{array}{l}\text { Findings in the physical } \\
\text { exploration }\end{array}$} \\
\hline Light touch sensitivity & 1 & 3.1 & - & - & - & - & - & . & - & - & . & - & - & - \\
\hline $\begin{array}{l}\text { Reduced in the } \\
\text { fingers }\end{array}$ & 1 & 3.1 & 0 & 0 & 0 & 0 & 1 & 100 & 0 & 0 & 0 & 0 & 0 & 0 \\
\hline Pin sensitivity & 0 & 0 & 0 & 0 & 0 & 0 & 0 & 0 & 0 & 0 & 0 & 0 & 0 & 0 \\
\hline Normal & 32 & 100 & 4 & 100 & 4 & 100 & 15 & 100 & 4 & 100 & 4 & 100 & 1 & 100 \\
\hline Vibratory sensitivity & 25 & 78.1 & - & - & & & - & - & - & - & - & - & - & - \\
\hline Normal & 7 & 21.9 & 1 & 25 & 1 & 25 & 2 & 13.3 & 1 & 25 & 2 & 50 & 0 & 0 \\
\hline $\begin{array}{l}\text { Reduced up to } \\
\text { wrist/ankle }\end{array}$ & 1 & 3.1 & 0 & 0 & 0 & 0 & 1 & 6.7 & 0 & 0 & 0 & 0 & 0 & 0 \\
\hline $\begin{array}{l}\text { Reduced up to } \\
\text { elbow/knee }\end{array}$ & 4 & 12.5 & 1 & 25 & 1 & 25 & 2 & 13.3 & 0 & 0 & 1 & 25 & 0 & 0 \\
\hline $\begin{array}{l}\text { Reduced above } \\
\text { elbow/knee }\end{array}$ & 20 & 62.5 & 2 & 50 & 2 & 50 & 10 & 66.7 & 3 & 75 & 1 & 25 & 1 & 100 \\
\hline $\begin{array}{l}\text { Right toe muscular } \\
\text { strength }\end{array}$ & 8 & 25 & . & . & . & . & . & . & - & . & . & . & . & . \\
\hline Normal & 24 & 75 & 4 & 100 & 3 & 75 & 12 & 80 & 3 & 75 & 2 & 50 & 0 & 0 \\
\hline $\begin{array}{l}\text { Lightly } \\
\text { decreased }\end{array}$ & 7 & 21.9 & 0 & 0 & 1 & 25 & 2 & 13.3 & 1 & 25 & 2 & 50 & 1 & 100 \\
\hline $\begin{array}{l}\text { Moderately } \\
\text { decreased }\end{array}$ & 1 & 3.1 & 0 & 0 & 0 & 0 & 1 & 6.7 & 0 & 0 & 0 & 0 & 0 & 0 \\
\hline $\begin{array}{l}\text { Left toe muscular } \\
\text { strength }\end{array}$ & 8 & 25 & . & . & . & . & - & . & - & . & . & . & . & . \\
\hline $\begin{array}{l}\text { Lightly } \\
\text { decreased }\end{array}$ & 8 & 25 & 0 & 0 & 1 & 100 & 5 & 100 & 1 & 100 & 1 & 100 & 0 & 0 \\
\hline $\begin{array}{l}\text { Right ankle muscular } \\
\text { strength }\end{array}$ & 11 & 34.3 & . & . & . & . & - & . & . & . & . & . & - & . \\
\hline Normal & 21 & 65.6 & 3 & 75 & 3 & 75 & 9 & 60 & 3 & 75 & 2 & 50 & 1 & 100 \\
\hline $\begin{array}{l}\text { Lightly } \\
\text { decreased }\end{array}$ & 9 & 28.1 & 1 & 25 & 1 & 25 & 4 & 26.7 & 1 & 25 & 2 & 50 & 0 & 0 \\
\hline
\end{tabular}




\begin{tabular}{|c|c|c|c|c|c|c|c|c|c|c|c|c|c|c|}
\hline $\begin{array}{l}\text { Severely } \\
\text { decreased }\end{array}$ & 2 & 6.3 & 0 & 0 & 0 & 0 & 2 & 13.3 & 0 & 0 & 0 & 0 & 0 & 0 \\
\hline $\begin{array}{l}\text { Left ankle muscular } \\
\text { strength }\end{array}$ & 11 & 34.3 & $\cdot$ & . & - & $\cdot$ & . & . & . & - & . & . & - & . \\
\hline $\begin{array}{l}\text { Lightly } \\
\text { decreased }\end{array}$ & 10 & 31.3 & 1 & 100 & 1 & 100 & 5 & 83 & 1 & 100 & 2 & 100 & 0 & 0 \\
\hline $\begin{array}{l}\text { Severely } \\
\text { decreased }\end{array}$ & 1 & 3.1 & 0 & 0 & 0 & 0 & 1 & 16.7 & 0 & 0 & 0 & 0 & 0 & 0 \\
\hline $\begin{array}{l}\text { Right phalanges } \\
\text { abduction muscular } \\
\text { strength }\end{array}$ & 7 & 21.9 & - & . & - & - & . & . & . & - & . & . & . & . \\
\hline $\begin{array}{l}\text { Lightly } \\
\text { decreased }\end{array}$ & 6 & 18.8 & 1 & 100 & 0 & 0 & 4 & 80 & 0 & 0 & 1 & 100 & 0 & 0 \\
\hline Paralysis & 1 & 3.1 & 0 & 0 & 0 & 0 & 1 & 20 & 0 & 0 & 0 & 0 & 0 & 0 \\
\hline $\begin{array}{l}\text { Left phalanges } \\
\text { abduction muscular } \\
\text { strength }\end{array}$ & 7 & 21.9 & . & . & . & . & . & . & . & . & . & . & . & . \\
\hline $\begin{array}{l}\text { Lightly } \\
\text { decreased }\end{array}$ & 6 & 18.8 & 1 & 100 & 0 & 0 & 4 & 80 & 0 & 0 & 1 & 100 & 0 & 0 \\
\hline Paralysis & 1 & 3.1 & 0 & 0 & 0 & 0 & 1 & 20 & 0 & 0 & 0 & 0 & 0 & 0 \\
\hline $\begin{array}{l}\text { Right wrist extension } \\
\text { muscular strength }\end{array}$ & 7 & 21.9 & - & . & . & - & . & - & . & . & . & . & . & . \\
\hline $\begin{array}{l}\text { Lightly } \\
\text { decreased }\end{array}$ & 6 & 18.8 & 1 & 100 & 0 & 0 & 3 & 75 & 1 & 100 & 1 & 100 & 0 & 0 \\
\hline Paralysis & 1 & 3.1 & 0 & 0 & 0 & 0 & 1 & 25 & 0 & 0 & 0 & 0 & 0 & 0 \\
\hline $\begin{array}{l}\text { Left wrist extension } \\
\text { muscular strength }\end{array}$ & 7 & 21.9 & - & . & . & - & . & . & . & . & . & . & . & . \\
\hline $\begin{array}{l}\text { Lightly } \\
\text { decreased }\end{array}$ & 6 & 18.8 & 1 & 100 & 0 & 0 & 3 & 75 & 1 & 100 & 1 & 100 & 0 & 0 \\
\hline Paralysis & 1 & 3.1 & 0 & 0 & 0 & 0 & 1 & 25 & 0 & 0 & 0 & 0 & 0 & 0 \\
\hline Right ankle deep reflex & 13 & 40.6 & - & - & - & - & - & - & - & - & - & - & - & - \\
\hline Normal & 19 & 59.4 & 2 & 50 & 2 & 50 & 7 & 46.7 & 3 & 75 & 4 & 100 & 1 & 100 \\
\hline Decreased & 10 & 31.3 & 1 & 25 & 1 & 25 & 7 & 46.7 & 1 & 25 & 0 & 0 & 0 & 0 \\
\hline Absent & 1 & 3.1 & 0 & 0 & 1 & 25 & 0 & 0 & 0 & 0 & 0 & 0 & 0 & 0 \\
\hline $\begin{array}{l}\text { All reflexes } \\
\text { absent }\end{array}$ & 2 & 6.3 & 1 & 25 & 0 & 0 & 1 & 6,7 & 0 & 0 & 0 & 0 & 0 & 0 \\
\hline Left ankle deep reflex & 13 & 40.6 & - & . & . & - & . & . & . & . & . & . & - & . \\
\hline Normal & 19 & 59.4 & 2 & 50 & 2 & 50 & 7 & 46.7 & 3 & 75 & 4 & 100 & 1 & 100 \\
\hline Decreased & 10 & 31.3 & 1 & 25 & 1 & 25 & 7 & 46.7 & 1 & 25 & 0 & 0 & 0 & 0 \\
\hline Absent & 1 & 3.1 & 0 & 0 & 1 & 25 & 0 & 0 & 0 & 0 & 0 & 0 & 0 & 0 \\
\hline $\begin{array}{l}\text { All reflexes } \\
\text { absent }\end{array}$ & 2 & 6.3 & 1 & 25 & 0 & 0 & 1 & 6.7 & 0 & 0 & 0 & 0 & 0 & 0 \\
\hline Right knee deep reflex & 6 & 18.8 & - & . & . & - & . & . & . & . & . & . & - & . \\
\hline Normal & 26 & 81.3 & 3 & 75 & 2 & 50 & 12 & 80 & 4 & 100 & 4 & 100 & 1 & 100 \\
\hline Decreased & 2 & 6.3 & 0 & 0 & 0 & 0 & 2 & 13.3 & 0 & 0 & 0 & 0 & 0 & 0 \\
\hline Absent & 2 & 6.3 & 0 & 0 & 2 & 50 & 0 & 0 & 0 & 0 & 0 & 0 & 0 & 0 \\
\hline $\begin{array}{l}\text { All reflexes } \\
\text { absent }\end{array}$ & 2 & 6.3 & 1 & 25 & 0 & 0 & 1 & 6.7 & 0 & 0 & 0 & 0 & 0 & 0 \\
\hline Left knee deep reflex & 6 & 18.8 & - & . & - & - & . & - & . & - & . & - & - & . \\
\hline Normal & 26 & 81.3 & 3 & 75 & 2 & 50 & 12 & 80 & 4 & 100 & 4 & 100 & 1 & 100 \\
\hline Decreased & 2 & 6.3 & 0 & 0 & 0 & 0 & 2 & 13.3 & 0 & 0 & 0 & 0 & 0 & 0 \\
\hline Absent & 2 & 6.3 & 0 & 0 & 2 & 50 & 0 & 0 & 0 & 0 & 0 & 0 & 0 & 0 \\
\hline $\begin{array}{l}\text { All reflexes } \\
\text { absent }\end{array}$ & 2 & 6.3 & 1 & 25 & 0 & 0 & 1 & 6.7 & 0 & 0 & 0 & 0 & 0 & 0 \\
\hline
\end{tabular}

